\title{
Condição de Saúde Bucal Autorreferida, Fatores Relacionados às Gestantes de Alto Risco
}

\author{
Suzely Adas Saliba Moimaz, ${ }^{1}$ Denise de Toledo Rós, ${ }^{2}$ \\ Aryane Kame Tamanaha, ${ }^{2}$ Nemre Adas Saliba, ${ }^{2}$ Tânia Adas Saliba ${ }^{2}$
}

\section{RESUMO}

O objetivo neste estudo foi avaliar o uso de serviço odontológico, condição de saúde bucal autorreferida por gestantes de alto risco e fatores relacionados. Foi realizada uma pesquisa transversal, tipo inquérito, com 1.200 gestantes de alto risco que realizaram o pré-natal em um centro de referência para atendimento especializado em saúde, em um período de dois anos. Foram excluídas aquelas com período gestacional avançado. Os dados foram processados no programa Bioestat 5.3, ao nível de significância de $5 \%$. As principais condições para classificação de gestação de alto risco foram: idade materna $(39,08 \%)$, hipertensão $(26,90 \%)$ e diabetes (10,32\%). Do total, 40,08\% realizaram sua última consulta odontológica há mais de 1 ano, estando o uso do serviço odontológico associado à ocupação da gestante $(p<0,02)$. Gestantes desempregadas tiveram 2,03 vezes mais chances do uso do serviço odontológico há menos de um ano ( $(\mathrm{n}=0,010)$. Entre as entrevistadas, $72,17 \%$ relataram a condição de saúde bucal de "regular" a "muito ruim" e $75,58 \%$ já haviam tido dor de origem dentária. A condição de saúde bucal autorreferida como regular/ruim/muito ruim foi relacionada com a idade, baixa escolaridade, baixa renda ( $p<0,0001)$, ocupação $(p<0,0001)$, estado civil $(p<0,0001)$ e com o uso do serviço há mais de 1 ano $(p<0,0001)$. Conclui-se que a maioria das gestantes considerou sua condição de saúde bucal "regular" ou "ruim/muito ruim", contudo a taxa de uso de serviços odontológicos foi baixa. A idade materna e obesidade foram as condições mais prevalentes para o alto risco gestacional.

Palavras-chave: assistência odontológica; diabetes gestacional; gravidez de alto risco; hipertensão induzida pela gravidez; saúde bucal.

\section{SELF-REPORTED ORAL HEALTH CONDITION, FACTORS RELATED TO HIGH-RISK PREGNANT WOMEN}

\section{ABSTRACT}

The aim of this study were to verify the use of dental care and oral health condition perception of high-risk pregnant women. This cross-sectional, inquiry-type research was performed with 1200 high-risk pregnant women who received prenatal care in a specialized health care reference center, in the period of two years. Women in advanced pregnancy and those who were not able to answer the questions were excluded. The data were analyzed in Epi Info 7.4.1 and Bioestat 5.3 software, at 5\% significance level. The most prevalent changes to the classification of high-risk pregnancies were maternal age (39.08\%), hypertension (26.90\%), and diabetes (10.32). From the total respondents, $40.08 \%$ had visited the dental office over 1 year ago, which was associated with age and occupation $(\mathrm{p}<0.002)$. Unemployed pregnant women were 2.03 times more likely to use the dental service less than a year ago $(p=0,010)$. From the respondents, $72.17 \%$ reported their teeth and gingiva as "fair" or "very poor", and $75.58 \%$ reported having had a toothache. The oral health condition perception was related to age $(p=0.0156)$, level of education $(p<0.0001)$, income $(p<0.0001)$, occupation $(p<0.0001)$, marital status $(p<0.0001)$, and dental care utilization $(p<0.0001)$. It can be concluded that the dental care utilization by high-risk pregnant women was still low, although a portion of the patients reported oral health as "fair" to "very poor". Maternal age and obesity were the most prevalent conditions for high gestational risk.

Keywords: dental care; diabetes gestacional; pregnancy high-risk; hypertension pregnancy-induced; oral health.

RECEBIDO EM: 22/9/2020

MODIFICAÇÕES SOLICITADAS EM: 4/1/2021

ACEITO EM: 1/7/2021

\footnotetext{
${ }^{1}$ Autora correspondente. Universidade Estadual Paulista Júlio de Mesquita Filho (Unesp) - Faculdade de Odontologia de Araçatuba, Departamento de Odontologia Infantil e Social. Rua José Bonifácio, 1.193 - Vila Mendonça. Araçatuba/SP, Brasil. CEP 16.015-050. http://lattes.cnpq.br/2799473073030693. https://orcid.org/0000-0002-4949-529X. suzely.moimaz@unesp.br

2 Universidade Estadual Paulista Júlio de Mesquita Filho (Unesp) - Faculdade de Odontologia de Araçatuba, Departamento de Odontologia Infantil e Social. Araçatuba/SP, Brasil.
} 


\section{INTRODUÇÃO}

A condição de saúde bucal pode influenciar a qualidade de vida dos indivíduos, afetando desde a mastigação e fonação até questões de origem psicológica, além de ser relacionada a alterações sistêmicas, como doença coronária cardíaca, diabetes e doenças renais (SERAPHIM et al., 2016; MUSSKOPF et al., 2018).

As alterações sistêmicas e outras afecções também podem influenciar no agravamento de condições bucais. A exemplo disto, Doenças Sexualmente Transmissíveis (DST), como a sífilis, podem desencadear o desenvolvimento alterado dos dentes do feto, e a Síndrome da Imunodeficiência Adquirida (Aids), predispor o surgimento da candidíase e lesões neoplásicas bucais, além de um maior risco ao desenvolvimento da cárie dentária, devido à diminuição do fluxo salivar, decorrente das medicações utilizadas no tratamento desta síndrome. Tratando-se da saúde periodontal, pode ser agravada em pacientes diabéticos, e em casos de má higienização dentária, que quando associado a alterações hormonais e traumas mecânicos, acarretam no desenvolvimento do granuloma piogênico (SERAPHIM et al., 2016; MOIMAZ et al., 2017a).

Em cada fase da vida, como na adolescência, na gestação e na velhice, a condição de saúde bucal pode sofrer mudanças devido às alterações físicas e psicológicas específicas de cada uma delas. Diante disso, não deve haver negligência nos cuidados à saúde bucal por parte dos serviços de saúde, priorizando o acesso às atividades de promoção à saúde e prevenção (ROVIDA et al., 2014).

O período gestacional é uma fase que merece atenção especial, devido às alterações de natureza hormonal, fisiológica, ambiental e psicológica. Em alguns casos, mulheres portadoras de enfermidades, condição física e/ou ambiental adversa ou que desenvolveram problemas neste período da vida, apresentam maiores probabilidades de evolução desfavorável, sendo classificadas como gestantes de alto risco (BRASIL, 2012). O descuido com a saúde bucal pode acarretar situações indesejadas na gestação. Nesse sentido, é de fundamental importância um acompanhamento especializado, com uma equipe multidisciplinar. Dessa forma, o cuidado odontológico pode ser realizado em qualquer período da gestação, com o segundo trimestre revelando-se o momento ideal, uma vez que a gestante se encontra em um quadro com menor prevalência de enjoos e o volume do útero não se encontra tão aumentado (VARGAS et al., 2016; MOIMAZ et al., 2017a).

De acordo com a orientação do Ministério da Saúde, as diferentes condições, consideradas na gestação de alto risco, podem ser classificadas em quatro categorias (BRASIL, 2012). A primeira categoria inclui as características pessoais ou sociodemográficas, abrangendo as condições relacionadas ao ambiente familiar inadequado, baixo nível educacional, exposição a agentes nocivos, violência doméstica, transtornos psicológicos, o uso de drogas ilícitas, idade materna, baixo peso da gestante. A segunda categoria são as morbidades como cardiopatias, hipertensão arterial prévia à gestação, câncer, epilepsia, ginecopatias, endocrinopatias como a diabetes mellitus, portadoras de doenças infecciosas como HIV, toxoplasmose e Doenças Sexualmente Transmissíveis (DSTs) e anemias. Na

Editora Unijuí - Revista Contexto \& Saúde - ISSN 2176-7114 - v. 21, n. 43, jul./set. 2021 
terceira categoria constam os antecedentes obstétricos incluindo abortos anteriores, cirurgias uterinas, infertilidade, multiparidade e o histórico de gestação anterior com alguma restrição de crescimento, prematuridade ou malformações. Na quarta categoria estão as doenças obstétricas na gestação atual que incluem malformações e óbito fetal, hipertensão e doenças endócrinas, aloimunização e ameaças de aborto (BRASIL, 2012; IPEA, 2014).

Elevados níveis de estrógeno e progesterona, o aumento da frequência de ingestão de alimentos, especialmente carboidratos, e náuseas, quando associados ao descuido da higiene bucal podem causar problemas bucais e/ou agravar doenças já existentes (FIGUEIREDO et al., 2017; YUNITA SARI; SADDKI; YUSOFF, 2020) A doença periodontal pode gerar dor, estresse e a disseminação bacteriana, consequentemente ocasionando prejuízos à gestação (SERAPHIM et al., 2016). Alguns estudos apontam a associação de complicações como parto prematuro, pré-eclâmpsia e recém-nascidos de baixo peso à doença periodontal, sem ainda evidências científicas comprovadas (SERAPHIM et al., 2016; IHEOZOR-EJIOFOR et al., 2017).

A gestação é uma fase favorável para a promoção de saúde bucal, uma vez que a mulher se encontra mais receptiva à novas informações e incorporação de hábitos positivos à sua saúde e consequentemente, de seus filhos (MOIMAZ et al., 2007; SILVA et al., 2020). No Brasil, a consulta odontológica foi inserida ao pré-natal, sendo reconhecida a importância da promoção e manutenção da saúde bucal das gestantes e a oferta do cuidado no SUS (BRASIL, 2017). Algumas barreiras devem ser ultrapassadas, como medo, insegurança, crenças e tabus sobre $\mathrm{o}$ atendimento odontológico durante a gestação, tanto por parte da gestante como dos profissionais da área (GEORGE et al., 2017; KATEEB; MOMANY, 2018; LIM et al., 2018; LIU et al., 2019).

Não foram encontrados na literatura estudos específicos sobre uso no serviço odontológico com gestantes de alto risco. Considerando a importância do cuidado à saúde da mulher, a escassez de pesquisas sobre gestação de alto risco e saúde bucal, e o fato de que, além das barreiras mencionadas, outros fatores complicadores podem dificultar o acesso de gestantes de alto risco ao cuidado e aos serviços assistenciais de saúde bucal, estudos sobre o tema devem ser realizados. Assim, os objetivos nesta pesquisa foram avaliar o uso do serviço odontológico, a condição de saúde bucal autorreferida e fatores relacionados às gestantes de alto risco.

\section{MÉTODOS}

Foi realizada pesquisa transversal, tipo inquérito, com 1.200 gestantes de alto risco atendidas em um centro de referência à atenção especializada do SUS no Estado de São Paulo, denominado Ambulatório Médico de Especialidades (AME). O AME selecionado é o local de referência de 28 municípios, prestando diversos serviços de média complexidade, entre eles atendimentos oftálmicos, nutricionais, dermatológicos, incluindo o atendimento obstétrico especializado para gestantes de alto risco.

Editora Unijuí - Revista Contexto \& Saúde - ISSN 2176-7114 - v. 21, n. 43, jul./set. 2021 


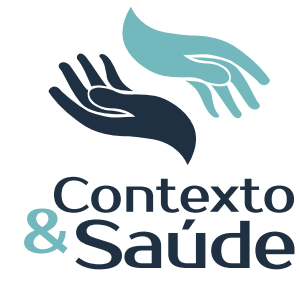

Para composição da amostra consideraram-se todas as gestantes de alto risco que realizaram o pré-natal no centro de referência e aceitaram participar da pesquisa no período de novembro de 2015 a novembro de 2017, totalizando 1.200 gestantes. Foram excluídas gestantes com avançado período gestacional ou que não possuíam condições físicas para participar da entrevista, devido a complicações gestacionais.

Um estudo-piloto foi realizado para teste e adequação do formulário da pesquisa e os seus resultados não foram incluídos neste estudo. Os dados foram coletados por meio de entrevistas estruturadas, realizadas em local isolado do serviço, empregando-se formulário específico para a pesquisa, que continha questões sobre o perfil sociodemográfico das gestantes, uso do serviço odontológico, amamentação, história médica e gestação atual das participantes. Para as questões socioeconômicas foi utilizado adaptação do formulário empregado no censo nacional, realizado pelo Instituto Brasileiro de Geografia e Estatística (IBGE, 2010)

As variáveis desfecho deste estudo foram: classificação da gestação de alto risco, uso do serviço odontológico e percepção sobre seus dentes e gengivas. As perguntas empregadas para verificação dessas variáveis foram: "Como a senhora acha que estão seus dentes e gengiva?", considerando como resposta uma escala de "muito ruim", "ruim", "regular", "bom", "muito bom", e "Quando foi sua última visita ao cirurgião-dentista?", considerando como respostas, "menos de 1 ano", "entre 1 e dois anos", "mais de 2 anos".

As variáveis independentes foram: idade ( $<20$ anos; 21 a 34 anos; $>34$ anos), renda familiar (até 1.500 reais; até 2.500 reais; mais de 2.500 reais; não sabe/não respondeu), escolaridade (até 7 anos; de 8 a 10 anos; 11 anos; mais de 11 anos), estado civil (solteira; casada; amasiada; divorciada/viúva), ocupação (autônoma; empregada; desempregada; do lar; estudante/pensionista), local de moradia (rural; urbano), semana gestacional (até 12 semanas; até 26 semanas; mais de 26 semanas), necessidade relatada de ir ao dentista (sim; não), relato de dor de origem odontológica (sim; não) e o local da última consulta odontológica (serviço público; particular; convênio; faculdade; outros; nunca foi ao dentista), motivo da consulta (revisão/prevenção; dor; tratamento no geral; tratamento ortodôntico; outros; nunca foi ao dentista) e a percepção sobre a última consulta odontológica (muito ruim; ruim; regular; bom; muito bom; nunca foi ao dentista).

Foram consideradas as frequências de cada condição de alto risco das gestantes e, posteriormente, categorizadas de acordo com os critérios descritos no manual "Gestação de Alto Risco: Manual Técnico", divulgado pelo Ministério da Saúde, em 2010 (BRASIL, 2012).

Os dados foram analisados e as distribuições de frequência absoluta e percentual das variáveis estudadas foram apresentadas por meio de tabelas. $O$ teste Qui-quadrado foi utilizado para verificar a associação do uso do serviço odontológico e da condição de saúde bucal autorreferida com as variáveis idade, renda, escolaridade, estado civil, ocupação, local de moradia e semana gestacional. Também foi analisada a associação entre o uso do serviço odontológico e a condição de saúde bucal autorreferida. Os dados foram processados com auxílio dos programas Epi Info versão 7.2 para Windows ${ }^{\circledR}$ e Bioestat 5.3 adotando-se um nível de significância de 5\%.

Editora Unijuí - Revista Contexto \& Saúde - ISSN 2176-7114 - v. 21, n. 43, jul./set. 2021 
Todas as participantes da pesquisa assinaram o Termo de Consentimento Livre e Esclarecido com detalhamento sobre os objetivos da pesquisa e a forma de divulgação dos dados. Esta pesquisa cumpriu todos os princípios éticos para pesquisas com seres humanos contidos na Declaração de Helsinki e no Código de Nuremberg e obteve a aprovação do Comitê de Ética em Pesquisa com Seres Humanos (Parecer número 1.914.629/2017).

Orientações sobre saúde bucal e sua relação com o aleitamento materno foram oferecidas para todas as gestantes. Quando identificada a necessidade de tratamento odontológico curativo, as gestantes foram encaminhadas para uma clínica de atenção especializada a esse público.

\section{RESULTADOS}

As principais condições e afecções que determinaram a classificação das gestantes como de "alto risco" estão apresentadas na Tabela 1. Nota-se que a idade materna foi a mais frequente das condições para o alto risco gestacional.

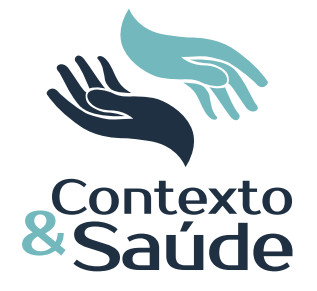

Tabela 1 - Condições mais prevalentes para o alto risco gestacional, Brasil, 2018

\begin{tabular}{|c|c|c|}
\hline Variáveis & & $\mathrm{n}(\%)$ \\
\hline \multirow{7}{*}{$\begin{array}{l}\text { Características } \\
\text { pessoais/ } \\
\text { sociodemográficas }\end{array}$} & Idade materna & $469(39,08)$ \\
\hline & Obesidade & $74(6,17)$ \\
\hline & Cirurgia anterior & $23(1,92)$ \\
\hline & Baixo peso & $7(0,58)$ \\
\hline & Transtornos mentais & $36(3,00)$ \\
\hline & Dependência de drogas lícitas/ilícitas & $106(8,83)$ \\
\hline & Deficiências físicas & $4(0,33)$ \\
\hline \multirow[t]{21}{*}{ Morbidades } & Hipertensão & $135(11.25)$ \\
\hline & Diabetes & $63(5,25)$ \\
\hline & Hipo/hipertireoidismo & $94(7,83)$ \\
\hline & Asma & $14(1,67)$ \\
\hline & Cardiopatia & $36(3,00)$ \\
\hline & Ginecopatias & $63(5,25)$ \\
\hline & Nefropatias & $13(1,08)$ \\
\hline & Toxoplasmose & $40(3,33)$ \\
\hline & Sífilis & $34(2,83)$ \\
\hline & Vírus da Imunodeficiência Adquirida (HIV) & $13(1,08)$ \\
\hline & Alterações vasculares/osteomusculares & $24(2,00)$ \\
\hline & Anemia & $38(3,17)$ \\
\hline & Papiloma Vírus Humano (HPV) & $18(1,50)$ \\
\hline & Lúpus & $6(0,50)$ \\
\hline & Trombofilias & $30(2,50)$ \\
\hline & Acidente Vascular Cerebral (AVC) & $5(0,42)$ \\
\hline & Câncer & $4(0,33)$ \\
\hline & Hérnia & $10(0,83)$ \\
\hline & Hanseníase & $2(0,17)$ \\
\hline & Desmaios/convulsão/epilepsia & $22(1,83)$ \\
\hline & Herpes & $3(0,25)$ \\
\hline
\end{tabular}

Editora Unijuí - Revista Contexto \& Saúde - ISSN 2176-7114 - v. 21, n. 43, jul./set. 2021 


\begin{tabular}{llr}
\hline Antecedentes & Abortamento habitual & $56(4,67)$ \\
obstétricos & Infertilidade & $5(0,42)$ \\
& Multiparidade & $7(0,58)$ \\
& Recém-nascido com restrições de crescimento, & $35(2,92)$ \\
& pré-termo ou malformado & $20(1,67)$ \\
\hline & Pré-eclâmpsia gestação anterior & $3(0,25)$ \\
\hline Doença obstétrica na & Aloimunização & $15(1,25)$ \\
gravidez atual & Amniorrexe prematura & $15(1,25)$ \\
& Desvio quanto ao crescimento uterino e & $37(3,08)$ \\
& volume de líquido amniótico & $63(5,25)$ \\
& Malformações fetais & $63(5,25)$ \\
& Gestação gemelar/trigemelar & $188(15,67)$ \\
& Síndromes hemorrágicas & $62(5,17)$ \\
\hline
\end{tabular}

Fonte: As autoras.

Do total de 1.200 gestantes, grande parte apresentava de 20 a 34 anos de idade $(61,08 \%)$, renda familiar inferior a 1.500 reais por mês $(47,58 \%), 84,75 \%$ possuíam até o Ensino Médio completo, 40,25\% eram casadas e 39,50\% estavam empregadas. Quase a totalidade $(96,92 \%)$ residia na zona urbana (Tabela 2 ). Houve associação significativa entre o uso do serviço odontológico e as variáveis idade $(p=0,0194)$ e ocupação $(p=0,0016)$.

Tabela 2 - Associação entre o uso do serviço odontológico e as variáveis sociodemográficas e outras características das gestantes de alto risco, Brasil, 2018

\begin{tabular}{|c|c|c|c|c|c|}
\hline & & \multicolumn{4}{|c|}{ Uso do serviço odontológico } \\
\hline & & $\begin{array}{l}\text { menos de um } \\
\text { ano }(n=719)\end{array}$ & $\begin{array}{l}\text { mais de um ano } \\
\qquad(n=481)\end{array}$ & $\begin{array}{c}\text { Total } \\
(n=1200)\end{array}$ & p valor* \\
\hline \multicolumn{2}{|l|}{ Variáveis } & $\mathrm{n}(\%)$ & $\mathrm{n}(\%)$ & $\mathrm{n}(\%)$ & \\
\hline & $<20$ anos & $135(18,78)$ & $67(13,94)$ & $202(16,83)$ & \\
\hline \multirow[t]{3}{*}{ Idade } & 20 a 34 anos & $417(58,00)$ & $316(65,70)$ & $733(61,08)$ & 0,0194 \\
\hline & $>34$ anos & $167(23,22)$ & $98(20,26)$ & $265(22,09)$ & \\
\hline & Até 1.500 reais & $338(47,01)$ & $233(48,44)$ & $571(47,58)$ & \\
\hline Renda & Até 2.500 reais & $227(31,57)$ & $167(34,72)$ & $394(32,84)$ & 0,1569 \\
\hline \multirow[t]{2}{*}{ Familiar } & $\begin{array}{l}\text { Mais de } 2.500 \\
\text { reais }\end{array}$ & $120(16,69)$ & $68(14,14)$ & $188(15,67)$ & \\
\hline & $\begin{array}{l}\text { Não sabe/não } \\
\text { respondeu }\end{array}$ & $34(4,73)$ & $13(2,70)$ & $47(3,91)$ & \\
\hline \multirow{4}{*}{ Escolaridade } & Até 7 anos & $118(16,41)$ & $92(19,13)$ & $210(17,51)$ & \\
\hline & De 8 a 10 anos & $221(30,74)$ & $152(31,60)$ & $373(31,08)$ & 0,5688 \\
\hline & 11 anos & $268(37,27)$ & $166(34,51)$ & $434(36,16)$ & \\
\hline & Mais de 11 anos & $112(15,58)$ & $71(14,76)$ & $183(15,25)$ & \\
\hline \multirow{4}{*}{ Estado civil } & Solteira & $160(22,25)$ & $86(17,88)$ & $246(20,50)$ & \\
\hline & Casada & $282(39,22)$ & $201(41,79)$ & $483(40,25)$ & 0,3167 \\
\hline & Amasiada & $258(35,88)$ & $179(37,21)$ & $437(36,42)$ & \\
\hline & Divorciada/viúva & $19(2,65)$ & $15(3,12)$ & $34(2,83)$ & \\
\hline \multirow[b]{3}{*}{ Ocupação } & Autônoma & $62(8,62)$ & $30(6,23)$ & $92(7,67)$ & \\
\hline & Empregada & $273(37,96)$ & $201(41,79)$ & $474(39,50)$ & \\
\hline & Desempregada & $122(16,97)$ & $76(15,80)$ & $198(16,50)$ & 0,0016 \\
\hline
\end{tabular}




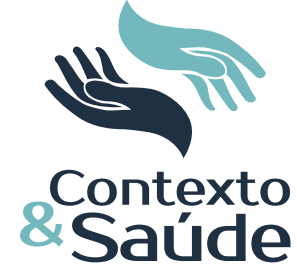

\begin{tabular}{|c|c|c|c|c|c|}
\hline & Do lar & $176(24,48)$ & $145(30,15)$ & $321(26,75)$ & \\
\hline & $\begin{array}{l}\text { Estudante/ } \\
\text { pensionista }\end{array}$ & $86(11,97)$ & $29(6,03)$ & $115(9,58)$ & \\
\hline Moradia & Rural & $24(3,34)$ & $13(2,70)$ & $37(3,08)$ & 0,6502 \\
\hline & Urbano & $695(96,66)$ & $468(97,30)$ & $1163(96,92)$ & \\
\hline Semana & Até 12 semanas & $84(11,68)$ & $47(9,78)$ & $131(10,91)$ & \\
\hline gestacional & Até 26 semanas & $356(49,51)$ & $227(47,19)$ & $583(48,59)$ & 0,2783 \\
\hline & $\begin{array}{l}\text { Mais de } 26 \\
\text { semanas }\end{array}$ & $279(38,80)$ & $207(43,03)$ & $486(40,50)$ & \\
\hline
\end{tabular}

Observa-se na Tabela 3 que grande parcela das gestantes (40,08\%) não havia feito uso de serviço odontológico no último ano. A maioria havia realizado sua última consulta odontológica no serviço público de saúde $(50,91 \%)$ e mais de um quarto dos motivos foi devido à dor.

Tabela 3 - Características da última consulta odontológica de gestantes de alto risco, Brasil, 2018

\begin{tabular}{|c|c|c|}
\hline Variáveis & & n (\%) \\
\hline \multirow[t]{4}{*}{ Última consulta odontológica } & Nunca & $12(1,00)$ \\
\hline & Menos de 1 ano & $719(59,92)$ \\
\hline & Entre 1 e 2 anos & $277(23,08)$ \\
\hline & Mais de 2 anos & $192(16,00)$ \\
\hline \multirow[t]{6}{*}{ Local da última consulta } & Serviço público & $589(49,08)$ \\
\hline & Particular & $473(39,42)$ \\
\hline & Convênio & $91(7,58)$ \\
\hline & Faculdade & $22(1,83)$ \\
\hline & Outros & $13(1,08)$ \\
\hline & Nunca foi ao dentista & $12(1,00)$ \\
\hline \multirow[t]{6}{*}{ Motivo da consulta } & Revisão/Prevenção & $303(25,25)$ \\
\hline & Dor & $319(26,58)$ \\
\hline & Tratamentos no geral & $317(26,42)$ \\
\hline & Tratamento ortodôntico & $181(15,08)$ \\
\hline & Outros & $68(5,67)$ \\
\hline & Nunca foi ao dentista & $12(1,00)$ \\
\hline \multirow{6}{*}{$\begin{array}{l}\text { Percepção sobre a última consulta } \\
\text { odontológica }\end{array}$} & Muito ruim & $37(3,08)$ \\
\hline & Ruim & $61(5,08)$ \\
\hline & Regular & $175(14,59)$ \\
\hline & Bom & $767(63,92)$ \\
\hline & Muito bom & $148(12,33)$ \\
\hline & Nunca foi ao dentista & $12(1,00)$ \\
\hline
\end{tabular}

Fonte: As autoras.

Conforme demonstrado na Tabela 4, houve associação significativa entre a condição de saúde bucal autorreferida como regular/ruim/muito ruim e as variáveis idade $(0,0156)$, renda familiar $(p<0,0001)$, escolaridade $(p<0,0001)$, estado civil $(p<0,0001)$ e ocupação $(p<0,0001)$. 
Tabela 4 - Associação entre a percepção de condição de saúde bucal e as variáveis sociodemográficas e outras características das gestantes de alto risco, Brasil, 2018

\begin{tabular}{|c|c|c|c|c|c|}
\hline & & \multicolumn{4}{|c|}{ Percepção sobre seus dentes e gengivas } \\
\hline \multirow[t]{2}{*}{ Variáveis } & & $\begin{array}{l}\text { Muito ruim / ruim } \\
\qquad(n=326)\end{array}$ & $\begin{array}{l}\text { Regular } \\
(n=540)\end{array}$ & $\begin{array}{l}\text { Bom / Muito } \\
\text { bom ( } n=334)\end{array}$ & \multirow[t]{2}{*}{$p$ valor ${ }^{*}$} \\
\hline & & $\mathrm{n}(\%)$ & $n(\%)$ & $\mathrm{n}(\%)$ & \\
\hline \multirow[t]{3}{*}{ Idade } & $<20$ anos & $38(11,66)$ & $107(19,82)$ & $57(17,07)$ & \multirow{3}{*}{0,0156} \\
\hline & 20 a 34 anos & $221(67,79)$ & $315(58,33)$ & $197(58,98)$ & \\
\hline & $>34$ anos & $67(20,55)$ & $118(21,85)$ & $80(23,95)$ & \\
\hline \multirow{4}{*}{$\begin{array}{l}\text { Renda } \\
\text { Familiar }\end{array}$} & Até 1.500 reais & $177(54,29)$ & $265(49,08)$ & $129(38,62)$ & \multirow{4}{*}{$<0,0001$} \\
\hline & Até 2.500 reais & $95(29,15)$ & $193(35,74)$ & $106(31,74)$ & \\
\hline & Mais de 2.500 reais & $39(11,96)$ & $62(11,48)$ & $87(26,05)$ & \\
\hline & $\begin{array}{l}\text { Não sabe/não } \\
\text { respondeu }\end{array}$ & $15(4,60)$ & $20(3,70)$ & $12(3,59)$ & \\
\hline \multirow{4}{*}{ Escolaridade } & Até 7 anos & $74(22,70)$ & $93(17,23)$ & $43(12,87)$ & \multirow{4}{*}{$<0,0001$} \\
\hline & De 8 a 10 anos & $104(31,90)$ & $182(33,70)$ & $87(26,05)$ & \\
\hline & 11 anos & $117(35,89)$ & $189(35,00)$ & $128(38,32)$ & \\
\hline & Mais de 11 anos & $31(9,51)$ & $76(14,07)$ & $76(22,76)$ & \\
\hline \multirow[t]{4}{*}{ Estado civil } & Solteira & $60(18,40)$ & $115(21,30)$ & $71(21,26)$ & \multirow{4}{*}{$<0,0001$} \\
\hline & Casada & $114(34,97)$ & $195(36,11)$ & $174(52,10)$ & \\
\hline & Amasiada & $141(43,25)$ & $214(39,63)$ & $82(24,55)$ & \\
\hline & Divorciada/viúva & $11(3,38)$ & $16(2,96)$ & $7(2,09)$ & \\
\hline \multirow[t]{5}{*}{ Ocupação } & Autônoma & $24(7,36)$ & $43(7,96)$ & $25(7,48)$ & \multirow{5}{*}{$<0,0001$} \\
\hline & Empregada & $114(34,97)$ & $209(38,70)$ & $151(45,21)$ & \\
\hline & Desempregada & $79(24,23)$ & $83(15,38)$ & $36(10,78)$ & \\
\hline & Do lar & $93(28,53)$ & $143(26,48)$ & $85(25,45)$ & \\
\hline & $\begin{array}{l}\text { Estudante/ } \\
\text { pensionista }\end{array}$ & $16(4,91)$ & $62(11,48)$ & $37(11,08)$ & \\
\hline \multirow[t]{2}{*}{ Moradia } & Rural & $12(3,68)$ & $16(2,96)$ & $9(2,69)$ & \multirow{2}{*}{0,7465} \\
\hline & Urbano & $314(96,32)$ & $524(97,04)$ & $325(97,31)$ & \\
\hline \multirow{3}{*}{$\begin{array}{l}\text { Semana } \\
\text { gestacional }\end{array}$} & Até 12 semanas & $35(10,74)$ & $63(11,67)$ & $33(9,88)$ & \multirow{3}{*}{0,9141} \\
\hline & Até 26 semanas & $157(48,16)$ & $258(47,78)$ & $168(50,30)$ & \\
\hline & $\begin{array}{l}\text { Mais de } 26 \\
\text { semanas }\end{array}$ & $134(41,10)$ & $219(40,55)$ & $133(39,82)$ & \\
\hline
\end{tabular}

Fonte: As autoras.

Gestantes que fizeram uso do serviço odontológico há menos de 1 ano relataram melhor condição de saúde bucal e menor necessidade de ir ao dentista. A frequência de gestantes que relataram dor de origem dentária foi elevada, e também demonstrou associação com o uso do serviço odontológico $(p<0,05)$ (Tabela 5). 
Tabela 5 - Associação entre o uso do serviço odontológico e a percepção de condição de saúde bucal das gestantes de alto risco, Brasil, 2018

\begin{tabular}{llrrr}
\hline & & \multicolumn{2}{c}{ Uso do serviço odontológico } & \\
\hline Variáveis & & \multicolumn{1}{c}{$\begin{array}{c}\text { Menos de um } \\
\text { ano }(\mathrm{n}=719)\end{array}$} & $\begin{array}{l}\text { Mais de um ano } \\
(\mathrm{n}=481)\end{array}$ & \multirow{2}{*}{ p valor* } \\
\cline { 2 - 4 } & & $\mathrm{n}(\%)$ & $\mathrm{n}(\%)$ & \\
\hline Percepção sobre seus & Muito ruim & $32(4,45)$ & $45(9,36)$ & $<0,0001$ \\
dentes e gengivas & Ruim & $125(17,39)$ & $124(25,78)$ & \\
& Regular & $324(45,06)$ & $216(44,90)$ & \\
& Bom & $228(31,71)$ & $87(18,09)$ & \\
& Muito bom & $10(1,39)$ & $9(1,87)$ & \\
\hline Acha que precisa ir ao & Sim & $571(79,42)$ & $444(92,31)$ & $<0,0001$ \\
dentista & Não & $148(20,58)$ & $37(7,69)$ & \\
\hline Já teve dor alguma vez & Sim & $567(78,86)$ & $340(70,69)$ & 0,0016 \\
& Não & $152(21,14)$ & $141(29,31)$ & \\
\hline Já teve dor de dente & Sim & $266(37,00)$ & $113(23,49)$ & $<0,0001$ \\
nos últimos 6 meses & Não & $453(63,00)$ & $368(76,51)$ & \\
\hline
\end{tabular}

*valores de p obtidos usando o teste de Qui-quadrado, nível de significância de $5 \%$

Fonte: As autoras.

\section{DISCUSSÃO}

As gestações de alto risco são assim classificadas por apresentarem alterações que podem levar prejuízos à mãe e/ou ao feto, como o surgimento ou agravamento de doença periodontal, pré-eclâmpsia e desfechos ruins no nascimento, como parto prematuro e baixo peso ao nascer (SILVA et al., 2020.). Neste estudo as condições de alto risco mais prevalentes foram a idade, hipertensão, diabetes, obesidade, dependência de drogas lícitas/ilícitas e distúrbios da tireoide.

O uso do serviço apresentou associação estatisticamente significante com a idade das gestantes, possivelmente devido ao fato de que grávidas mais jovens apresentam menor necessidade de tratamento restaurador ou reabilitador ou até mesmo por estas apresentarem maior conhecimento sobre a importância do atendimento odontológico durante a gestação. Resultado semelhante foi encontrado no estudo de Saliba et al. (2020), no qual a maior procura por atendimento odontológico deu-se por gestantes mais velhas. Este fato pode estar relacionado aos mitos e tabus existentes acerca da saúde bucal e atendimento odontológico durante a gestação, dado que, erroneamente, o sangramento gengival é considerado normal, ou ainda devido ao medo de gestantes em consultar o cirurgião-dentista. A adolescência, por si só, não é considerada fator de risco para a gestação, todavia trata-se de um período de transição na vida da mulher, podendo acarretar um risco psicossocial devido à imaturidade emocional e à aceitação da gestação. Muitas adolescentes também tendem a buscar novas experiências, e assim, podem iniciar o uso de drogas lícitas e ilícitas. Tais mudanças psicológicas e comportamentais podem trazer consequências à condição de saúde bucal, especialmente quando a higiene bucal é negligenciada (BRASIL, 2012; ROVIDA et al., 2014; MOIMAZ et al., 2017a).

Editora Unijuí - Revista Contexto \& Saúde - ISSN 2176-7114 - v. 21, n. 43, jul./set. 2021 


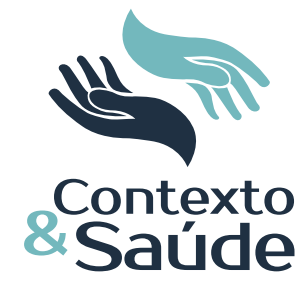

No caso de gestantes com idade mais avançada e/ou obesas existe um aumento no risco de desenvolvimento de malformações fetais e de doenças sistêmicas durante a gestação, como a hipertensão e o diabetes gestacional (MOIMAZ et al., 2017a). O diabetes é uma enfermidade que vem sendo relacionada diretamente com a doença periodontal, devido ao aumento da resistência à insulina em decorrência do processo inflamatório existente (MOIMAZ et al., 2017a). Um estudo longitudinal verificou que gestantes com baixa escolaridade e obesidade tiveram filhos com maiores chances de desenvolver cárie dentária aos 5 anos, considerando que as mães introduziam uma dieta rica em açúcares no início da vida da criança (WIGEN; WANG, 2011).

Apesar da baixa frequência neste estudo, as DSTs exigem grande atenção devido à possiblidade de transmissão da doença da mãe para o filho, a transmissão vertical, durante a gestação ou parto, acarretando em sequelas para o bebê como: aborto, parto prematuro, herpes neonatal e sífilis congênita. A presença de lesões fundamentais na boca, como úlceras e nódulos, e as doenças bucais como candidíase e leucoplasia, são importantes sinais para o diagnóstico precoce das DSTs, associados à análise de exames sorológicos. Algumas DSTs, como a sífilis, também podem ocasionar alterações no desenvolvimento oral do bebê, como mudanças no formato e posicionamento dos dentes e o aparecimento de lesões ulcerativas (MOIMAZ et al., 2017a). Desta forma, evidencia-se a importância de ações e estratégias de saúde voltadas para a gestação de alto risco, visando a diminuir os riscos no desenvolvimento da gestação, que podem gerar sequelas tanto para a mãe como para o bebê.

Nesta pesquisa foi verificado que o uso do serviço odontológico por gestantes de alto risco foi baixo. Salienta-se que não foram encontrados na literatura estudos sobre as taxas do uso do serviço odontológico por gestantes de alto risco. Em pesquisas realizadas, a demanda de gestantes, em geral, por serviços odontológicos variou de 29\% a 42\% (MARCHI et al., 2019; YUNITA SARI; SADDKI; YUSOFF, 2020).

O uso do serviço odontológico, nesta pesquisa, esteve associado à ocupação. As gestantes desempregadas e estudantes/pensionistas, tiveram duas vezes mais chances de terem feito uso de serviços odontológicos há menos de um ano. Sugere-se que estas gestantes possuem maior tempo livre para cuidar de si e de sua saúde, havendo então uma maior procura pelo serviço por parte destas gestantes. Diversas barreiras podem ser apontadas para explicar as baixas taxas de atendimento odontológico durante a gestação. Entre elas destacam-se: a percepção sobre os problemas bucais, o tempo de espera para o atendimento, medo e insegurança com relação ao tratamento, falta de orientação sobre os efeitos da condição de saúde bucal para a gestação e a resistência ao atendimento pelo cirurgião-dentista (RIGGS et al., 2016; GEORGE et al., 2017; KATEEB; MOMANY, 2018; LIM et al., 2018; LIU et al., 2019).

Aproximadamente metade das gestantes entrevistadas realizaram consulta odontológica no serviço público de saúde e uma parcela relevante considerou de "muito ruim" a "regular" o seu último atendimento odontológico. A experiência anterior negativa quanto ao serviço odontológico pode ser considerada uma barreira ao acesso para atenção à saúde bucal. Vários podem ser os motivos

Editora Unijuí - Revista Contexto \& Saúde - ISSN 2176-7114 - v. 21, n. 43, jul./set. 2021 


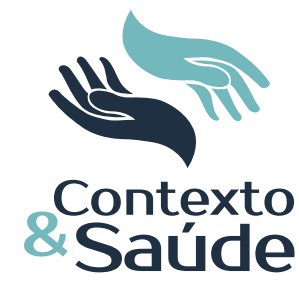

relacionados à insatisfação, entre eles a falta de resolutividade do serviço, filas de espera para atendimento, dor ou problema ocorrido durante o tratamento (MOIMAZ et al., 2017b; SALIBA et al., 2018). Em contrapartida, estudos sobre percepção do usuário acerca do atendimento odontológico no SUS mostraram resultados positivos em relação ao atendimento odontológico oferecido (MOIMAZ et al., 2015a, 2015b). Evidencia-se que a avaliação dos serviços de saúde por parte da população é de fundamental importância para a compreensão das barreiras de acesso e resolutividade dos serviços.

A dor foi um dos principais motivos para o não uso dos serviços odontológicos entre as gestantes e esteve associada com a procura recente pelo atendimento. Este achado é corroborado por outros estudos (LIU et al., 2019; YUNITA SARI; SADDKI; YUSOFF, 2020) e é de extrema importância, visto que a dor pode interferir negativamente na gestação e poderia ser evitada com consultas preventivas. Os atendimentos de urgência são mais dispendiosos e sobrecarregam os serviços de saúde quando comparados com as atividades preventivas e de promoção de saúde, que atingem maiores parcelas da população e são mais efetivas e de menor custo (MOIMAZ et al., 2007).

No Brasil, os serviços de saúde pública são organizados segundo os níveis de Atenção: Primária, Secundária e Terciária. A Atenção Primária é composta pelas Unidades de Saúde, que são a porta de entrada para os serviços preventivos ou curativos, atividades clínicas de baixa densidade tecnológica, não emergenciais e as atividades de saúde pública. O pré-natal de baixo risco é realizado na Atenção Primária à saúde (LAVRAS, 2011). Procedimentos que necessitam de profissionais especializados, de maior complexidade para sua resolução, são referenciados para a Atenção Secundária. No caso do pré-natal, quando identificada alguma alteração que possa acarretar agravos à saúde da mãe ou do bebê, a paciente é referenciada para um serviço especializado para a realização do pré-natal de alto risco (LAVRAS, 2011; SALIBA et al., 2013). Convém salientar que a Unidade de Saúde continua tendo vínculo com essa paciente e, no caso da confirmação da gestação de alto risco, o pré-natal é acompanhado também pela Unidade de Saúde. Diante do exposto, é de grande importância que haja integração entre os níveis de atenção primária, secundária e terciária para garantia da eficácia e resolutividade dos serviços e integralidade do cuidado em saúde.

A maioria das gestantes relatou que apresentava condição de saúde bucal de "muito ruim" a "regular". Neste contexto, em estudo similar realizado com gestantes atendidas em uma instituição pública, verificou-se que mesmo relatando a necessidade de tratamento dentário, o atendimento odontológico não era entendido como prioridade para elas (SALIBA et al., 2020). Gestantes com menor renda, baixa escolaridade e desempregadas relataram piores condições bucais. Esse fato evidencia que as condições sociais interferem diretamente nas condições de saúde e mesmo em situação de baixa escolaridade as necessidades de saúde são sentidas pelas pessoas (LIM et al., 2018; SILVA et al., 2020).

O fato de a percepção de saúde bucal estar intimamente relacionada à imagem que a paciente tem dela própria, e não vinculada necessariamente àquilo que se apresenta clinicamente, pode ser uma limitação, devido à metodologia aplicada no estudo. Nesse sentido, destaca-se a importância da percepção da

Editora Unijuí - Revista Contexto \& Saúde - ISSN 2176-7114 - v. 21, n. 43, jul./set. 2021 


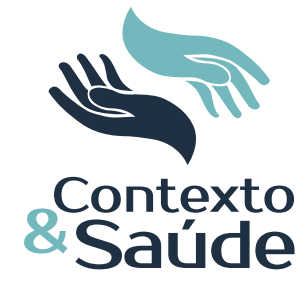

condição de saúde bucal para a busca do serviço odontológico. É importante que esta procura, contudo, não seja apenas para procedimentos curativos e/ou de emergência, mas de métodos preventivos, para a manutenção de uma condição de saúde bucal saudável.

Neste estudo houve associação entre o uso recente do serviço odontológico e a percepção da condição de saúde bucal, sendo esta considerada um fator importante para a procura pelo serviço, visto que gestantes que tiveram sua última consulta odontológica há mais de um ano relataram percepções negativas sobre sua condição de saúde bucal. A percepção de não possuir nenhum problema bucal pode ser considerada uma barreira ao atendimento odontológico na gestação (ROCHA et al., 2018; MARCHI et al., 2019).

A demanda nos serviços odontológicos por gestantes é uma questão complexa e de fundamental importância. Estudos demonstraram que as gestantes, mesmo acreditando que a gravidez poderia causar alterações bucais, não procuravam atendimento odontológico (RIGGS et al., 2016; LORENZO-POUSO et al., 2018). Apesar de as pesquisas sobre o atendimento odontológico para grávidas terem ganhado destaque nos últimos anos, não foram encontrados na literatura estudos em relação ao uso no serviço odontológico pelas gestantes de alto risco. Embora este tema esteja em expansão na área da saúde, as barreiras com relação ao atendimento ainda persistem, mostrando-se necessário maior empenho por parte da equipe multiprofissional que realiza o pré-natal para desmistificar o atendimento odontológico na gestação. A atenção odontológica para gestantes de alto risco é indispensável para a promoção da saúde.

\section{CONCLUSÕES}

A maioria das gestantes considerou sua condição de saúde bucal "regular" ou "ruim/muito ruim", contudo a taxa de uso de serviços odontológicos foi baixa. A idade materna e obesidade foram as condições mais prevalentes para o alto risco gestacional.

\section{AGRADECIMENTOS}

O presente trabalho foi realizado com apoio da Coordenação de Aperfeiçoamento de Pessoal de Nível Superior - Brasil (Capes) - Código de Financiamento 001.

\section{REFERÊNCIAS}

BRASIL. Ministério da Saúde. Secretaria de Atenção à Saúde. Departamento de Ações Programáticas Estratégicas. Gestação de alto risco: manual técnico. 5. ed. Brasília: Ministério da Saúde, 2012.

BRASIL. Ministério da Saúde. Secretaria de Atenção à Saúde. Departamento de Atenção Básica. PMAQ: Programa de Melhoria do Acesso e da Qualidade: manual instrutivo para as equipes de Atenção Básica e Nasf. Brasília: Ministério da Saúde; 2017.

FIGUEIREDO, C. S. A. et al. Systemic alterations and their oral manifestations in pregnant women. Journal of Obstetrics and Gynaecology Research, v. 43, n. 1, p. 16-22, 2017.

Editora Unijuí - Revista Contexto \& Saúde - ISSN 2176-7114 - v. 21, n. 43, jul./set. 2021 
GEORGE, A. et al. Knowledge, attitude and practises of dentists towards oral health care during pregnancy: a cross sectional survey in New South Wales, Australia. Australian Dental Journal, v. 62, n. 3, p. 301-310, 2017.

IHEOZOR-EJIOFOR, Z. et al. Treating periodontal disease for preventing adverse birth outcomes in pregnant women. The Cochrane Database of Systematic Reviews, v. 6, n. 6, p. CD005297, 2017.

IBGE. Instituto Brasileiro de Geografia e Estatística. Censo 2010. 2010. Disponível em: https://censo2010.ibge.gov.br/coleta/questionarios.html. Acesso em: 20 jul. 2020.

IPEA. Instituto de Pesquisa Econômica Aplicada. Objetivos de desenvolvimento do milênio: relatório nacional de acompanhamento. Brasília: Ipea, 2014. Disponível em: https:// www.ipea.gov.br/portal/index.php?option=com_content $\& v i e w=a r t i c l e \& i d=22538$. Acesso em: 20 jul. 2020.

KATEEB, E.; MOMANY, E. Factors related to high dental caries experience in Palestinian pregnant women in the Jerusalem governorate: a cross-sectional study. Lancet, v. 391, sup. 2, p. S11, 2018.

LAVRAS, C. Atenção primária à saúde e a organização de redes regionais de atenção à saúde no Brasil. Saúde e Sociedade, v. 20, n. 4, p. 867-874, 2011.

LIM, M. et al. Midwives' and women's views on accessing dental care during pregnancy: an Australian qualitative study. Australian Dental Journal, v. 63, n. 3, p. 320-328, 2018.

LIU, P. P. et al. Dental care-seeking and information acquisition during pregnancy: a qualitative study. International Journal of Environmental Research and Public Health, v. 16, n. 14, p. 2.621, 2019.

LORENZO-POUSO, A. I. et al. Self-assessment of opinions, habits and oral health status by pregnant women in the south of Galicia, Spain. Semergen, v. 44, n. 2, p. 138-143, 2018.

MARCHI, K. S. et al. Medical provider promotion of oral health and women's receipt of dental care during pregnancy. Maternal and Child Health Journal, v. 23, n. 7, p. 890-902, 2019.

MOIMAZ, S. A. S. et al. O acesso de gestantes ao tratamento odontológico. Revista de Odontologia da Universidade Cidade de São Paulo, v. 18, n. 1, p. 39-45, 2007.

MOIMAZ, S. A. S. et al. O cidadão usuário do serviço odontológico do Sistema Único de Saúde sabe onde reclamar? O controle social na saúde. Journal of the Health Sciences Institute, v. 33, n. 4, p. 323-327, 2015a.

MOIMAZ, S. A. S. et al. Satisfação e perfil de usuários do serviço odontológico no Sistema Único de Saúde. RFO UPF, v. 20, n. 3, p. 334-339, 2015b.

MOIMAZ, S. A. S. et al. Aspectos da saúde geral e bucal de gestantes de alto risco: revisão da literatura. Journal of the Health Sciences Institute, v. 35, n. 3, p. 223-230, 2017a.

MOIMAZ, S. A. S. et al. Satisfação dos usuários segundo variáveis de organização dos serviços públicos odontológicos. Archives of Health Investigation, v. 6, n. 1, p. 14-19, 2017b.

MUSSKOPF, M. L. et al. Oral health related quality of life among pregnant women: a randomized controlled trial. Brazilian Oral Research, v. 32, p. e002, 2018.

RIGGS, E. et al. "We are all scared for the baby": promoting access to dental services for refugee background women during pregnancy. BMC Pregnancy and Childbirth, v. 16, n. 1, p. 12, 2016.

ROCHA, J. S. et al. Barriers and facilitators to dental care during pregnancy: a systematic review and meta-synthesis of qualitative studies. Cadernos de Saúde Pública, v. 34, n. 8, p. e00130817, 2018.

ROVIDA, T. A. S. et al. Self-perception and oral health in pregnant adolescents. Oral Health and Dental Management, v. 13, n. 3, p. 842-846, 2014.

SALIBA, N. A. et al. Organization of the demand for a Centre of Dental Specialties. Revista de Odontologia da Unesp, v. 42, n. 5, p. 317-323, 2013.

SALIBA, T. A. et al. Satisfação com atendimento e intenção de amamentação de gestantes em uma instituição pública. Archives of Health Investigation, v. 7, n. 2, p. 54-58, 2018.

SALIBA, T. A. et al. Associação entre percepção de saúde bucal e procura pelo serviço odontológico por gestantes. Saúde e Desenvolvimento Humano, v. 8, n. 1, p. 77-84, 2020.

Editora Unijuí - Revista Contexto \& Saúde - ISSN 2176-7114 - v. 21, n. 43, jul./set. 2021 
SERAPHIM, A. P. C. G. et al. Relationship among periodontal disease, insulin resistance, salivary cortisol, and stress levels during pregnancy. Brazilian Dental Journal, v. 27, n. 2, p. 123-127, 2016.

SILVA, C. C. et al. Acesso e utilização de serviços odontológicos por gestantes: revisão integrativa de literatura. Ciência \& Saúde Coletiva, v. 25, p. 827-835, 2020.

VARGAS, P. L. et al. Atenção qualificada ao pré-natal e puerpério: Uma intervenção em serviço na atenção básica. Revista Contexto \& Saúde, v. 16, n. 31, p. 4-14, 2016.

WIGEN, T. I.; WANG, N. J. Maternal health and lifestyle, and caries experience in preschool children: a longitudinal study from pregnancy to age 5yr. European Journal of Oral Science, v. 119, n. 6, p. 463-468, 2011.

YUNITA SARI, E.; SADDKI, N.; YUSOFF, A. Association between perceived oral symptoms and presence of clinically diagnosed oral diseases in a sample of pregnant women in Malaysia. International Journal of Environmental Research and Public Health, v. 17, n. 19, p. 7.337, 2020. 